\title{
Impact of External Cue Validity on Driving Performance in Parkinson's Disease
}

\author{
Karen Scally, ${ }^{1}$ Judith L. Charlton, ${ }^{2}$ Robert Iansek, ${ }^{3}$ John L. Bradshaw, ${ }^{1}$ Simon Moss, ${ }^{4}$ \\ and Nellie Georgiou-Karistianis ${ }^{1}$
}

\author{
${ }^{1}$ Experimental Neuropsychology Research Unit, School of Psychology and Psychiatry, Faculty of Medicine, Nursing \\ and Health Sciences, Monash University, Clayton Campus, VIC 3800, Australia \\ ${ }^{2}$ Monash Accident Research Centre, Monash University, Clayton Campus, VIC 3800, Australia \\ ${ }^{3}$ Clinical Research Centre for Movement Disorders and Gait, Kingston Centre, Warrigal Road, Cheltenham, \\ VIC 3192, Australia \\ ${ }^{4}$ School of Psychology, Deakin University, 221 Burwood Highway, Burwood, VIC 3125, Australia
}

Correspondence should be addressed to Nellie Georgiou-Karistianis, nellie.georgiou-karistianis@monash.edu

Received 15 November 2010; Accepted 27 March 2011

Academic Editor: Irena Rektorova

Copyright (C) 2011 Karen Scally et al. This is an open access article distributed under the Creative Commons Attribution License, which permits unrestricted use, distribution, and reproduction in any medium, provided the original work is properly cited.

\begin{abstract}
This study sought to investigate the impact of external cue validity on simulated driving performance in 19 Parkinson's disease (PD) patients and 19 healthy age-matched controls. Braking points and distance between deceleration point and braking point were analysed for red traffic signals preceded either by Valid Cues (correctly predicting signal), Invalid Cues (incorrectly predicting signal), and No Cues. Results showed that PD drivers braked significantly later and travelled significantly further between deceleration and braking points compared with controls for Invalid and No-Cue conditions. No significant group differences were observed for driving performance in response to Valid Cues. The benefit of Valid Cues relative to Invalid Cues and No Cues was significantly greater for PD drivers compared with controls. Trail Making Test (B-A) scores correlated with driving performance for PDs only. These results highlight the importance of external cues and higher cognitive functioning for driving performance in mild to moderate PD.
\end{abstract}

\section{Introduction}

In addition to its cardinal motor signs (bradykinesia, postural instability, resting tremor, cogwheel rigidity), a distinctive profile of cognitive deficits has been well documented in Parkinson's disease (PD) [1-10]. Although primarily dysexecutive in origin, cognitive impairment has been observed across a range of domains including attention, working memory, verbal and visual memory, visuoperception, visuospatial functioning, verbal fluency, planning, and organizational abilities [11-17].

PD has been characterised by a particular deficit in the volitional control or internal cueing of movement, with patients typically demonstrating significantly slower initiation and execution times as well as reduced accuracy of movement compared to age-matched controls [18-22]. This internal cueing deficit is also present on tasks that primarily demand cognition rather than motor performance, with impairment observed in the ability to use advance information to internally cue responses on Stroop colour naming and cognitive set-shifting tasks [23, 24]. Importantly, research findings have further demonstrated that provision of valid external cues compensates for defective internal initiation and improves performance on both motor and cognitive tasks in PD relative to controls [19, 20, 25-35]. Although invalid cues-providing incorrect information about the task requirements- have been found to prolong response times in neurologically normal subjects, the impact of invalid cues on performance in PD has generated conflicting results: some studies have reported greater performance costs in PD relative to controls [35-39], whereas other studies have found the opposite pattern of results or no group differences $[25,40,41]$. These inconsistent findings may be ascribed to 
differences across studies particularly in the timing of the invalid cue relative to the contradictory task demand.

Because of the range of motor and cognitive impairments observed in $\mathrm{PD}$, the potential impact on driving performance has become a topical issue with critical implications for safety $[42,43]$. Driving is a time-pressured activity that imposes constant demand on an individual's attentional resources, requiring simultaneous processing of different stimuli, prompt responding to environmental cues, anticipation of potential hazards, and the ability to plan and execute movement in a continually changing environment [44-46].

Investigations of on-road driving ability in PD have consistently reported significantly higher incidence of atfault safety errors for patients relative to controls [4754]. The type of errors most commonly reported across studies involved visual scanning and checking behaviours prior to lane changing and pulling out into traffic as well as unspecified difficulties negotiating T-intersections, traffic light intersections, and roundabouts $[48,50,51]$. More recent research has further highlighted particular areas of difficulty during on-road driving performance in PD. Uc et al. [54] found that PD drivers exhibited significant difficulty visually scanning and verbally reporting landmarks and road signs compared with controls. Moreover, in another study, PD drivers committed significantly more incorrect turns and became lost more often than control drivers during a routefinding task [55].

Simulator studies have also documented significant deficits in specific driving skills of PD drivers relative to age-matched controls, including delayed reaction time in response to both traffic signal change $[56,57]$ as well as simple auditory and visual cues [58]. Similarly, PD drivers demonstrate a reduced ability to stop at red lights [56, 57], poor detection of imminent collisions [59], and have significantly more collisions [60].

Stolwyk et al. [57] further investigated the impact of internal and external cues on simulated driving performance. Results indicated that, consistent with numerous previous findings of dependence on external cues to generate action, drivers with PD relied heavily on late-occurring external cues to initiate driving responses at traffic signals even when they had acquired internal knowledge of the upcoming events. When external cueing was unavailable, driving performance of the control group significantly benefitted from internalised advance information. In contrast, PDs were unable to utilise internal knowledge to improve driving performance in the absence of external cues. The findings of this study indicate that a persistent dependence on the external environment to guide driving behaviours, in addition to welldocumented prolonged reaction times, could compromise safe driving in Parkinson's disease, generating important implications for driving assessment outcomes such as the use of licence restrictions to limit driving to a familiar locale.

Given the dependence of PD drivers on external guidance for initiating driving responses, even when anticipatory action is possible and safer, the utilisation of external cues during driving warrants further investigation. Driving occurs in a continually changing environment with many dynamic cues that can change quickly and unpredictably and may be valid, invalid, or contradictory. For example, a green light, but a pedestrian crossing against signal represents a contradictory cue. Drivers must be able to adapt and change to a new course of action quickly, depending on the environmental demands.

The research on driving in PD to date has primarily utilised driving scenarios with clear and predictable task demands. One study [57] showed that due to impaired internal cueing, PD drivers were overreliant on external prewarning cues to initiate driving responses, but it remains unknown how PD drivers respond to a variety of dynamic cues that are more representative of the range of task demands in real driving. The purpose of the current study was to investigate driving performance of $\mathrm{PD}$ drivers and healthy controls in response to Valid Cues, Invalid Cues, and No Cues. Examining the impact of cue validity on driving performance will give some insight into PD driver's ability to respond to changing task demands. It will also reveal the potential costs and benefits of this overreliance on external cues to guide driving behaviour.

Because the sole driving event utilised was a traffic signal, the driving performance variables of interest were approach speed, braking point, and deceleration-to-brake point distance in response to red traffic signals preceded either by No Cue, a Valid Cue (Correct), or an Invalid Cue (Incorrect), which advised participants of the upcoming traffic signal phase (red or green). Only red signal events were analysed, because green lights did not necessitate a braking response. Other driving performance measures such as deceleration point, stopping point, and mean speed were not selected for analysis because of poor sensitivity for this particular experiment.

It was hypothesised that patients would brake significantly later than controls in the Invalid and No Cue conditions. It was further hypothesised that patients' mean braking point would benefit to a greater extent from Valid Cues relative to No Cues, whereas controls would demonstrate less difference between their braking points under Valid and No Cue conditions. It was hypothesized that Invalid Cues would produce later mean braking points relative to the other cue conditions for both groups although specific predictions were not formed about group differences in the response cost of Invalid Cues. Finally, it was predicted that patients would demonstrate greater deceleration to braking point distance across all cueing conditions compared to controls.

\section{Method}

2.1. Participants. Nineteen mild to moderately affected individuals with $\mathrm{PD}$ voluntarily participated. PD participants consisted of 4 females and 15 males whose age ranged from $52-81$ years $(M=68.74, \mathrm{SD}=6.72)$. All patients were clinically diagnosed by a neurologist (R.I.) with age at onset ranging from 51 to 77 years $(M=62.32$, SD $=$ $8.19)$ and disease duration ranging from 1 year to 17 years $(M=6.58, \mathrm{SD}=4.51)$. PD motor symptom severity was assessed using the motor subscale of the Unified Parkinson's Disease Rating Scale (UPDRS: scores ranged from 0 to 37, 
$M=15.37, \mathrm{SD}=9.86)$. All PD participants were on an established levodopa medication regime (Madopar, Sinemet or Stalevo) and were tested in the morning when they were optimally medicated. In addition, 5 participants were also on agonist medication (Cabergoline/Dostinex) and another 5 patients were on comt Inhibitors (Tasmar or Comtan). Nineteen matched, neurologically healthy control subjects also participated. Control participants consisted of 6 females and 13 males whose age ranged from 56 to 78 years $(M=$ $68.05, \mathrm{SD}=7.20)$. There was no statistical difference in age between the two groups, $t(36)=.30, P>.05$.

All participants held a valid driver's license and were driving on a regular basis - at least once a week. A brief interview and screening tests were administered to ensure that no participant demonstrated any uncorrected visual or hearing impairment or any history of debilitating physical conditions, drug or alcohol dependence, psychiatric illness, dementia, or head injury. All participants scored 23 or above on the Mini-Mental State Examination (MMSE) [61] and no significant difference was noted between PDs $(M=$ $29.21, \mathrm{SD}=1.23)$ and controls $(M=29.37, \mathrm{SD}=1.12)$, $t(36)=-.42, P>.05$. Depressive symptoms were evaluated using the Montgomery and Asberg Depression Rating Scale (MADRS). No significant difference was found in MADRS scores between PDs $(M=3.37, \mathrm{SD}=3.02)$ and controls $(M=2.58, \mathrm{SD}=2.61), t(36)=.86, P>.05$. Moreover, there was no significant difference in level of education (years) between patients $(M=14.16, \mathrm{SD}=3.06)$ and controls $(M=13.63, \mathrm{SD}=4.32), t(36)=.43, P>.05$. Also, no significant difference was found in the number of years of driving experience between the $\mathrm{PD}(M=48.74, \mathrm{SD}=7.13)$ and control groups $(M=49.11, \mathrm{SD}=7.84), t(36)=-.15$, $P>.05$.

No participants reported taking any medication known to impair driving performance, other than the medications used to treat PD symptoms. Table 1 contains a summary of group characteristics and neurocognitive scores. The study was approved by the Monash University Human Ethics Committee, and written informed consent was obtained from all participants.

2.2. Procedure. All participants completed testing in one session of approximately 2.5 hours duration at the Monash University Accident Research Centre (MUARC). Screening tasks were then completed (MMSE and MADRS), followed by a written questionnaire regarding demographics and driving history. Prior to commencing the experimental driving scenarios, participants were informed about all aspects of participation including the possibility of experiencing motion sickness, basic mechanics and capabilities of the simulator, breakdown of the testing session, process for communication during testing, and procedures for discontinuation. Participants then completed the baseline Current Well-Being Questionnaire in which they rated the degree of motion sickness symptoms currently present.

Participants first undertook a familiarisation drive for approximately 3 minutes in which the investigator sat in the passenger seat and explained the simulator controls including steering, acceleration, and braking. A practice drive was then undertaken by participants alone but with two-way communication available between control room and the car. Participants were allowed to practice until they reported that they felt competent and comfortable enough with the simulator controls to proceed to the first experimental task (usually around 5-10 minutes). The experimental driving scenario was split into two drives, each approximately 15 minutes. Participants were granted rest breaks in between these drives to minimise fatigue and simulator discomfort. Participants were also monitored for signs of simulator discomfort during the driving tasks via a camera mounted on the dashboard. If participants displayed any of the signs, such as increased swallowing, licking lips, yawning or sweating, they were advised to stop the driving task immediately.

The experiment consisted of a straight arterial drive with 26 traffic signals in total across the two drives (13 Red; 13 Green). The speed limit was set at $50 \mathrm{~km} / \mathrm{hr}$ for this experiment to minimise the risk of simulator sickness as a consequence of the frequent stopping in the task. On approach to each traffic signal, participants either encountered no warning cue or one of two Warning Cues informing them about the phase of the upcoming traffic signal: Red or Green. The Warning Cue utilised was a modified version of the "Prepare to Stop" signal used on Australian roads, in which flashing amber lights indicate that the imminent traffic light is about to change from Green to Red. The warning cue was placed 70 metres prior to the traffic signal in accordance with its real world use. However, the sign was altered slightly for this experiment. Instead of amber lights that flashed or did not flash, the sign utilised flashing red lights to indicate a change to Red and flashing green lights to indicate an upcoming Green light. Therefore, the Warning Cue was either Valid (congruent with traffic signal) or Invalid (incongruent with traffic signal). Intersections were placed approximately 700 metres apart to allow participants time to accelerate up to $50 \mathrm{~km} / \mathrm{hr}$ before approaching the next intersection. A diagram of the driving event is shown in Figure 1

Participants were advised of the starting speed limit for the drive and instructed to pay attention to their speed, remain within $10 \mathrm{~km}$ of the speed limit, and respond as appropriate to traffic signals throughout the drive. At the end of the second drive, participants completed the questionnaire to reassess well-being.

\subsection{Apparatus}

2.3.1. Driving Simulator Performance. The MUARC Advanced Driving Simulator consists of a Silicon Graphics Indy (for development and running of driving scenarios), a Silicon Graphics Onyx (for graphics generation, vehicle data inputs and outputs, control of audio system and vehicle dynamics), and a personal computer (for generating sounds). The simulator interface comprises a GM Holden sedan with normal interior appearance and controls; a curved projection screen in front of the vehicle, which provides a 180 degree field of view; a quadratic sound system producing realistic 
TABLE 1: Means (and SDs) of demographic and neurocognitive information for PD and control groups.

\begin{tabular}{|c|c|c|c|c|}
\hline \multirow[b]{2}{*}{ Age at testing (yrs) } & \multicolumn{2}{|c|}{$\begin{array}{c}\mathrm{PD} \\
(n=19)\end{array}$} & \multicolumn{2}{|c|}{$\begin{array}{l}\text { Control } \\
(n=19)\end{array}$} \\
\hline & 68.74 & $(6.72)$ & 68.05 & $(7.20)$ \\
\hline Years since diagnosis & 6.74 & $(4.51)$ & - & - \\
\hline Age at disease onset & 62.58 & $(8.19)$ & - & - \\
\hline UPDRS motor score & 15.37 & $(9.86)$ & - & - \\
\hline Driving experience (yrs) & 48.74 & $(7.13)$ & 49.11 & $(7.84)$ \\
\hline Education (yrs) & 14.16 & $(3.06)$ & 13.63 & $(4.32)$ \\
\hline MMSE score $(\max 30)$ & 29.21 & $(1.23)$ & 29.37 & $(1.12)$ \\
\hline MADRS score $(\max 60)$ & 3.37 & $(3.02)$ & 2.58 & $(2.61)$ \\
\hline Digit Span (SS: WMS-III) & 11.74 & $(3.38)$ & 12.42 & $(2.71)$ \\
\hline Mental Control (SS: WMS-III) & 12.21 & $(2.88)$ & 13.42 & $(2.29)$ \\
\hline Trails A (time in seconds) & 43.05 & $(20.20)$ & 35.74 & $(11.56)$ \\
\hline Trails B (time in seconds) & $106.16^{*}$ & $(63.76)$ & 73.63 & $(24.63)$ \\
\hline Trails B-A (time in seconds) & $63.11^{*}$ & $(50.44)$ & 37.89 & $(20.26)$ \\
\hline Hayling (SS) & 4.42 & $(1.77)$ & 5.21 & $(1.81)$ \\
\hline Brixton (SS) & 4.74 & $(2.13)$ & 5.84 & $(1.50)$ \\
\hline
\end{tabular}

UPDRS: Unified Parkinson's Disease Rating Scale (Max score 108); MMSE: Mini Mental State Exam (Max score 30, cutoff score 23); MADRS: Montgomery and Asberg Depression Rating Scale (Max score 60); Trails B-A: Score derived from subtraction of score for Trails A from Trails B; SS: Scaled score; WMS-III: Wechsler Memory Scale—Third Edition. *: Sig Group difference at 0.05 level.

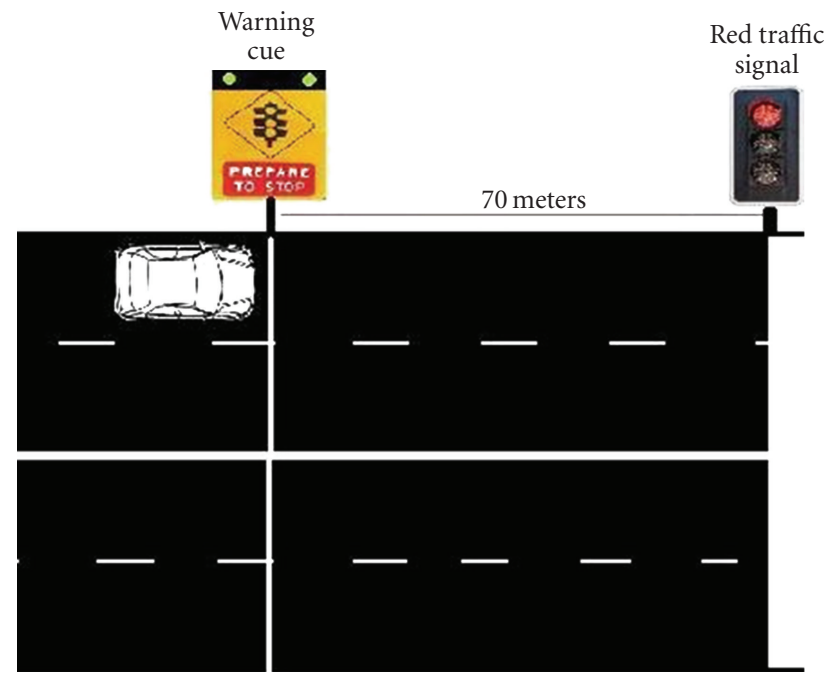

Trigger point for traffic signal change

Figure 1: Example of the Cueing Driving Event. Warning cue was either Valid (red flashing lights) or Invalid (green flashing lights).

traffic sounds and low-frequency vibrations; and a motion platform underneath the vehicle to simulate the feel of the road and allow up-down movement, and pitch and roll rotations. The MUARC Advanced Driving Simulator has been validated against on-road driving for research on speedrelated variables [62].

2.3.2. Data Analysis. Group means of the driving performance variables (approach speed, deceleration point, braking point) were calculated for each of the red light signals across each of the three Cueing conditions (Valid, Invalid, and No Cue). All statistical comparisons were conducted on these group means using various Analysis of Variance (ANOVA) techniques. A series of Pearson's product moment correlations were also performed to examine associations between driving performance measures and scores on clinical indices and screening tasks. One-way MANOVAs were used to compare groups on approach speed and braking point. The relative distance of deceleration point and braking point was compared across groups within each of the cueing conditions using one-way ANCOVAs.

\section{Results}

Means and standard deviations of approach speed, deceleration point, and braking point for each of the cueing conditions are presented in Table 2.

\subsection{Differences in Driving Performance across Groups within Cueing Conditions}

3.1.1. Approach Speed. Mean approach speeds indicate that both patients (Valid $M$ 48.30 SD 4.86; Invalid $M 47.85$ SD 5.25; No-Cue $M 47.19$ SD 5.42) and controls (Valid $M 48.88$ SD 2.69; Invalid $M$ 50.67 SD 3.76; No-Cue $M 49.39$ SD 2.91) were able to maintain a steady speed across the cueing conditions and remain within the speed limit $(50 \mathrm{~km} / \mathrm{hr})$ as instructed. No statistically significant difference was found in approach speed between groups for any of the cueing conditions (Valid-Cue, $(1,36)=0.20, P=.66$; Invalid-Cue, $F(1,36)=3.64, P=.07$; No-Cue, $F(1,36)=2.43, P=.13)$.

3.1.2. Braking Points. Both patients and controls braked within 70 metres of the traffic signal when no cue was 
TABLE 2: Red traffic signal means (and SDs) of driving variables for each group across the three cueing conditions.

\begin{tabular}{lccccccc}
\hline & & \multicolumn{3}{c}{ Valid } & \multicolumn{2}{c}{ Cueing Condition } \\
Driving Variable & Group & \multicolumn{2}{c}{ Invalid } & \multicolumn{2}{c}{ No Cue } \\
\hline \multirow{2}{*}{ Approach Speed $(\mathrm{km} / \mathrm{h})$} & PD & 48.30 & $(4.86)$ & 47.85 & $(5.25)$ & 47.19 & $(5.42)$ \\
& Control & 48.88 & $(.901)$ & 50.67 & $(3.76)$ & 49.39 & $(2.91)$ \\
Deceleration Point (m to signal) & PD & 117.70 & $(15.19)$ & 119.79 & $(18.96)$ & 101.76 & $(22.84)$ \\
& Control & 119.20 & $(24.41)$ & 113.92 & $(34.09)$ & 107.54 & $(30.76)$ \\
Brake Point (m to signal) & PD & 62.56 & $(18.21)$ & 50.64 & $(18.99)$ & 41.65 & $(9.64)$ \\
& Control & 72.52 & $(33.89)$ & 74.56 & $(39.10)$ & 61.66 & $(35.37)$ \\
Decel to Brake Pt Distance $(\mathrm{m})(\mathrm{se})$ & PD & 62.10 & $(5.64)$ & 51.36 & $(7.00)$ & 40.30 & $(5.28)$ \\
& Control & 72.98 & $(5.64)$ & 73.85 & $(7.00)$ & 63.02 & $(5.28)$ \\
\hline
\end{tabular}

present. For the Valid Cue condition, in which the cue correctly predicted a red signal, the difference in braking points between patients $(M=62.56, \mathrm{SD}=18.21)$ and controls $(M=72.52, \mathrm{SD}=33.89)$ was not statistically significant, $F(1,36)=1.86, P=.181$. However, for the Invalid Cue condition, in which the cue incorrectly predicted a green signal, patients with PD $(M=50.64, \mathrm{SD}=18.99)$ braked significantly later than did controls $(M=74.56$, $\mathrm{SD}=39.10), F(1,36)=6.95, P=.012$. Likewise, in the NoCue condition, patients with $\mathrm{PD}(M=41.65, \mathrm{SD}=9.64)$ also braked significantly later than did controls $(M=61.66$, $\mathrm{SD}=35.37), F(1,36)=12.81, P=.001$.

\subsection{Effect of Cues on Braking Performance for Each Group.} For each group, the effect of cueing condition on braking points was examined. Analyses for the control group found no significant main effect of cue type on braking point (Valid $M=72.52, \mathrm{SD}=33.89$; Invalid $M=74.56, \mathrm{SD}=39.10$; No Cue $M=61.66, \mathrm{SD}=35.37)$. Conversely, analyses for the PD group did find a significant main effect of cue type on braking point, $F(2)=12.56, P=.00$. The results of post hoc tests showed a significant difference between patients' braking points under Valid $(M=62.56, \mathrm{SD}=18.21)$ and No Cue $(M=41.65, \mathrm{SD}=9.64)$ conditions $(M$ diff $=-20.91$, S.E. $=3.66, P=.00)$. Moreover, a significant difference was also found between patients' braking points for Valid $(M=$ $62.56, \mathrm{SD}=18.21)$ and Invalid $(M=50.64, \mathrm{SD}=18.99)$ cue conditions $(M$ diff $=11.92$, S.E. $=3.90, P=.02)$. However, the mean difference between patients' braking points under Invalid Cue and No Cue conditions $(M$ diff $=-8.99$, S.E. $=$ 4.89) was not statistically significant $(P=.23)$.

\subsection{Relative Distance between Deceleration Point and Braking} Point. The relative points at which deceleration and braking occurred were compared between the groups for each of the cueing conditions. Separate ANCOVAs were conducted for each condition, with braking points designated as the dependent variables and corresponding deceleration point designated as the covariates. Under Valid Cue conditions, mean braking point for patients $(M=62.56, \mathrm{SD}=18.21)$ occurred on average 55.14 meters after their first deceleration point $(M=117.70, \mathrm{SD}=15.19)$, whereas braking point for controls $(M=119.20, \mathrm{SD}=24.41)$ occurred on average 46.68 meters after their first deceleration point $(M=72.52$,
$\mathrm{SD}=33.89)$. The distance between deceleration and braking points for Valid Cue conditions did not differ significantly between patients and controls, $F(1,36)=1.859, P=.18$.

Under Invalid Cue conditions, patients' mean braking point $(M=50.64, \mathrm{SD}=18.99)$ occurred 69.15 meters after their initial deceleration point $(M=119.79, \mathrm{SD}=18.96)$, whereas controls' mean braking point $(M=74.56$, SD $=$ 39.10) occurred just 39.36 meters after their first deceleration point $(M=113.92, \mathrm{SD}=34.09)$. Statistical analysis revealed that the distance between deceleration and braking points for Invalid Cue conditions was significantly greater in patients than in the controls, $F(1,36)=5.134, P=.03$.

Finally, for No-Cue conditions, braking point in the patient group $(M=41.65, \mathrm{SD}=9.64)$ occurred 60.11 meters after initial deceleration point $(M=101.76$, SD 22.84). In contrast, braking point for the control group $(M=61.66$, $\mathrm{SD}=35.37)$ was 45.88 meters after their initial deceleration point $(M=107.54, \mathrm{SD}=30.76)$. The difference between deceleration and braking points under No-Cue conditions was significantly greater in the PDs compared to the controls, $F(1,36)=9.21, P=.005$.

3.4. Correlations between Driving Performance and Cognitive and Clinical Indices. Correlations between driving variables and neurocognitive measures for the patient and control groups are presented in Table 3 .

The screening tasks and neurocognitive measures that were found to differ between groups were correlated with the driving performance variables, namely, braking point and deceleration-to-brake point distance for each cueing condition. Results of correlations for the PD group will be discussed first. UPDRS motor scores and MADRS scores did not significantly correlate with any of the driving performance variables under any of the Cueing conditions in the PD group. However, it was found that patients who scored better on the MMSE tended to brake later in the absence of external cues $(r=-.50, P<.05)$. Scores on part A of the Trail Making Test (TMT-A), a measure of psychomotor speed involving focused attention, visual scanning, and motor planning, were positively correlated with earlier braking point during invalid cue conditions $(r=.60, P<.01)$ for the PD group: slower psychomotor speed was therefore associated with earlier braking in response to invalid cues. Likewise, completion time on part B of the Trail Making Test 
TABLE 3: Correlations between driving performance variables and Trail Making Test scores (part B and B-A).

\begin{tabular}{|c|c|c|c|c|}
\hline \multirow{2}{*}{ Driving performance measures } & \multicolumn{2}{|c|}{ Trails B } & \multicolumn{2}{|c|}{ Trails B-A } \\
\hline & $\mathrm{PD}$ & Control & $\mathrm{PD}$ & Control \\
\hline $\begin{array}{l}\text { Valid Cues } \\
\text { Braking Point (m) }\end{array}$ & .288 & $.581^{* *}$ & .197 & .379 \\
\hline $\begin{array}{l}\text { Invalid Cues } \\
\text { Braking Point (m) }\end{array}$ & $.606^{* *}$ & $.592^{* *}$ & $.524^{*}$ & .435 \\
\hline $\begin{array}{l}\text { No Cues } \\
\text { Braking Point }(\mathrm{m})\end{array}$ & -.152 & .373 & -.163 & .211 \\
\hline $\begin{array}{l}\text { Valid Cues } \\
\text { Deceleration to Brake } \\
\text { Point Distance }(\mathrm{m})\end{array}$ & -.092 & $-.541^{*}$ & -.014 & -.357 \\
\hline $\begin{array}{l}\text { Invalid Cues } \\
\text { Deceleration to Brake } \\
\text { Point Distance }(\mathrm{m})\end{array}$ & -.399 & -.432 & -.337 & -.281 \\
\hline $\begin{array}{l}\text { No Cues } \\
\text { Deceleration to Brake } \\
\text { Point Distance }(\mathrm{m})\end{array}$ & .414 & -.215 & .351 & -.071 \\
\hline
\end{tabular}

(TMT-B), which imposes the same psychomotor demands as TMT-A with an additional working memory and setswitching component, also showed a significant positive correlation with invalidly cued braking point $(r=.61$, $P<.01)$. This indicates that poorer psychomotor speed and set-shifting ability was associated with earlier braking under invalid cue conditions. Moreover, TMT (B-A), which reflects the attention, and set-switching component of TMT$B$, independent of psychomotor speed, showed a positive correlation with invalidly cued braking point $(r=.52, P<$ $.05)$, indicating that with the contribution of psychomotor speed removed, poorer attention and set-switching was associated with earlier braking in response to invalid cues.

In the control group, scores on the MADRS and MMSE did not correlate with any driving performance measures. However, TMT-A performance was positively correlated with braking point under valid $(r=.58, P<.05)$ and invalid $(r=$ $.50, P<.05)$ cue conditions and was negatively correlated with deceleration to brake point distance for valid cues $(r=$ $-.54, P<.05)$. This indicates that slower psychomotor speed was associated with earlier braking under valid and invalid cue conditions and shorter distance travelled between deceleration and braking points in response to valid cues. Similarly, TMT-B was also positively correlated with braking point for valid cues $(r=.58, P<.01)$ and invalid cues $(r=.59, P<.01)$ and negatively correlated with deceleration to brake point distance under valid cue conditions $(r=-.54$, $P<.05)$. Together these indicate that slower psychomotor speed and attention switching abilities were related to earlier braking points under valid and invalid cue conditions and shorter distance travelled between deceleration and brake point when validly cued. However, with the psychomotor speed component removed, TMT (B-A) failed to correlate with any driving performance variables in the control group.

\section{Discussion}

This study sought to further investigate cue utilization by examining the impact of cue validity on simulated driving performance in PD. External cue validity was manipulated to examine how PD drivers adjust to different and changing environmental cue demands, reflecting the dynamic nature of the real driving environment. Approach speed, braking point, and deceleration-to-brake point distance were evaluated in response to three Cueing conditions throughout the simulated drive: Valid Cueing, Invalid Cueing, and No Cueing. We also explored correlations between the driving performance measures found to be significantly different between groups and scores on neurocognitive measures. It was first hypothesised that patients would brake significantly later than controls for both the Invalid and No Cue conditions. Consistent with this prediction, patients were found to brake significantly later than controls under Invalid and No-Cue conditions, yet braked comparably to controls under Valid Cue conditions. This pattern of findings reinforces the purported benefits of valid external cues on motor and cognitive performance in $\operatorname{PD}[19,20,24-29,31-35,63]$.

Importantly, under both Cueing conditions, the warning Cue was placed 70 metres prior to the traffic light, consistent with VicRoads regulations for the State of Victoria, Australia. Moreover, traffic signal change was triggered by driver presence at the 70 meter mark across all conditions, at which point the light then changed from green, to amber followed by red over a two-second timeframe. Thus, any braking points occurring before the 70 meter mark could not have been informed by traffic signal change, although the traffic light itself was minimally visible through a fog screen from approximately 200 meters and easily visible within 100 meters. Accordingly, although control participants initiated braking a few meters prior to passing the Cue 
under both Valid and Invalid Cueing conditions, patients braked eight meters beyond the Cue during Valid conditions and 20 meters beyond the Cue during Invalid conditions. This observation implies that, although controls' braking responses reflected anticipatory action in response to the warning Cue, patients' braking responses showed delayed initiation, occurring after both the warning Cue and the onset of traffic signal change. Nevertheless, the finding that PD patients braked earlier under Valid Cue conditions compared to Invalid Cue conditions, and in Cued conditions compared to No-Cue conditions, indicates that the presence of cues and, particularly Valid Cues, improved driving performance perhaps by triggering preparatory motor action and thereby facilitating earlier braking responses. This supposition accords with previous research, showing that control drivers were able to internally generate deceleration and braking responses prior to the display of external cues, whereas PD drivers were dependent on the appearance of late occurring external cues to initiate driving responses [57].

Our second hypothesis predicted a greater facilitatory effect of Valid Cues relative to No Cues on mean braking point for patients compared with controls. Consistent with this prediction, patients benefitted to a significantly greater extent ( $20.91 \mathrm{~m}$ gain in brake point) than controls ( $10.86 \mathrm{~m}$ gain) from the provision of Valid Cues relative to noncued conditions. This finding is also consistent with results from peripheral cueing tasks that report greater effects of Valid cueing for patients compared with controls [41].

We further hypothesised that Invalid Cues would elicit significantly later braking points for PDs and Controls relative to braking points under Valid and No-Cue conditions. While patients' braking points showed a significant advantage in response to Valid Cues relative to Invalid Cues, controls were found to brake similarly during Valid and Invalid Cueing conditions. This result contrasts to findings of studies using central cueing paradigms that reported similar effects of Valid Cueing relative to Invalid Cueing on task performance of patients and age-matched controls [41] and may simply reflect the relatively low demands of the driving task which did not sufficiently challenge the control participants. Interestingly, results further indicated that, compared with the No Cue condition, Invalid Cues did not incur any significant response cost to braking point for either group. Indeed compared to the No Cue condition, braking points were similarly facilitated by the presence of Invalid Cues with an 8.99 meter advantage noted for patients and 12.9 meter advantage found for controls. This benefit contradicts typical findings from cueing studies utilizing various experimental paradigms and likely reflects the limitations of driving simulator studies to sufficiently replicate the cognitive demands and experimental control of discrete psychophysical tasks within a simulated drive that alone presents a challenging trade-off between maximizing ecological validity, participant well-being and experimental control. Alternatively, the mere presence of the flashing light may have a high level of salience for driving safety, and therefore even the invalid cues may have augmented arousal, facilitating performance on this functional task.
Our final hypothesis concerned deceleration to brake point distance which is measured in meters but essentially reflects the time elapsed between the initial point of deceleration on approach to the warning sign and the affirmative action of applying the brake pedal. We predicted that deceleration to brake point distance would be significantly greater for patients relative to controls across all cueing conditions. Consistent with the results for braking point, but against expectations, no significant difference was observed between the groups in deceleration-to-brake point distance for Valid Cues. In contrast, and in line with our predictions, deceleration-to-brake point distance was significantly greater for patients compared with controls under both Invalid Cue and No Cue conditions. This measure essentially reflects a combination of decision and movement time between the point drivers initially began to decelerate on approach to the warning sign and the point they actually applied the brake, although unfortunately this measure cannot be parsed into the separate component processes.

Nevertheless, although the time course of approach to the intersection at the outset, as represented by approach speed and deceleration point, was similar in patients and controls, as the event drew closer, driving performance in these groups began to diverge, except when Valid Cues were provided. This pattern of observations is consistent with previous findings of significantly delayed reaction time on various tasks in $\mathrm{PD}$, particularly under circumstances of increased complexity: a delay that is successfully ameliorated by Valid Cues. Determining the extent to which decision time and reaction time contribute to driving performance output measures will have important implications for clinical estimation of driver safety particularly with regard to hazard perception and time-to-collision judgments. Theoretically, given the evidence of impairments in visuoperceptual and visuospatial processes, attention and executive functions, delayed responses are likely to reflect inefficiencies in cognitive information processing that informs responses, in addition to slowness in initiating the motor response itself. Indeed, performances on both Trails A and Trails B were significantly positively correlated with invalidly cued braking point in both patients and controls, such that slower psychomotor speed and set-shifting abilities corresponded with earlier braking point in both groups. Moreover, driving performance remained significantly positively correlated with TMT (B-A), a measure of cognitive set switching with the psychomotor speed component removed, in patients only, further highlighting the pertinence of information processing. Surprisingly, higher scores on the MMSE were significantly negatively correlated with braking point under noncued conditions in the patient group only indicating that superior cognitive ability was associated with later braking in the absence of external cues. Collectively, these findings suggest that perhaps those with poorer cognitive functioning and/or cognitive inflexibility adopt a more cautious driving style as a compensatory mechanism.

The significant correlations between driving performance and scores on the Trail Making Test are consistent with those of previous PD driving studies [48, 54, 55, 64, 65] although such studies rarely report significant correlations 
with the MMSE and typically implicate several independent areas of cognition, visual, and/or motor function as potential contributors to impaired driving ability in PD. Such functions include but are not limited to contrast sensitivity [47, $66,67]$, information processing speed $[49,67]$, visuospatial and planning abilities $[47,48,65]$, attention $[54,55]$, motor dexterity $[48,50]$, and both visual and verbal memory [49, $50,55]$. The general lack of consensus in the literature as to the particular functions likely to impact on driving ability in PD may reflect differences in the driving measures used for correlation and in the size and disease characteristics of the samples utilized across studies. It may also be indicative of an inherent difficulty in identifying independent predictors of impaired functioning on a multifactorial task within a heterogenous clinical population. Nevertheless, in contrast to previous studies, with one notable exception [59], the current sample of PD drivers performed comparatively to controls on all cognitive tasks except for the Trail-Making Test perhaps highlighting the importance of higher cognitive information processing and set-shifting abilities for driving performance in the mild to moderate stages of $\mathrm{PD}$. While the Trial Making Test seems to have a unique value in correlating with driving performance measures, further research with larger samples and perhaps clinical subtypes of PD is required to determine whether this test is a suitable screening tool for fitness to drive. Moreover, such a study would need to go beyond correlational analyses and be designed specifically for predictive analyses in order to address issues of sensitivity and specificity.

There are many advantages to using a driving simulator, including increased safety, greater experimental control, and reduced costs; however, it should be borne in mind that simulated driving performance does not fully equate to on-road driving performance. In addition, a relatively small sample size was utilised in this study. Future research should employ larger sample sizes with greater variation on disease indices to explore the effects of PD heterogeneity and associated cognitive function on driving performance. The current results are particularly noteworthy given the largely comparable cognitive functioning of the two groups and despite the relatively low demands of the simulated driving task which utilized a conservative speed limit $(50 \mathrm{~km} / \mathrm{hr})$, straight road, simple visual environment and use of a single driving event that differed only in terms of the cue and response requirement. Hence, contrary to the general consensus in the PD literature that cognitive and motor difficulties are most likely to arise in complex and cognitively demanding situations; it has been shown that patients may experience difficulties with driving even at low speeds in which rapid reactions times are not as crucial. Overall, the present results indicate that patients drive significantly worse than healthy age-matched controls under both noncued and invalidly cued conditions and that driving performance is significantly improved with provision of valid warning cues. It is anticipated that these and future results will assist in developing compensatory strategies and thereby improving driver safety for individuals with PD.

\section{References}

[1] B. Dubois and B. Pillon, "Cognitive deficits in Parkinson's disease," Journal of Neurology, vol. 244, no. 1, pp. 2-8, 1997.

[2] S. A. Raskin, J. C. Borod, and J. Tweedy, "Neuropsychological aspects of Parkinson's disease," Neuropsychology Review, vol. 1, no. 3, pp. 185-221, 1990.

[3] E. Martignoni and D. Calandrella, "Mental manifestations in Parkinson's disease," Minerva Psichiatrica, vol. 50, no. 1, pp. 27-44, 2009.

[4] B. E. Levin and H. L. Katzen, "Early cognitive changes and nondementing behavioral abnormalities in Parkinson's disease," in Behavioral Neurology of Movement Disorders, pp. 85-95, Raven Press, New York, NY, USA, 1995.

[5] I. Bodis-Wollner, "Neuropsychological and perceptual defects in Parkinson's disease," Parkinsonism and Related Disorders, vol. 9, supplement 5, pp. S83-S89, 2003.

[6] D. J. Zgaljardic, J. C. Borod, N. S. Foldi, and P. Mattis, "A review of the cognitive and behavioral sequelae of Parkinson's disease: relationship to frontostriatal circuitry," Cognitive and Behavioral Neurology, vol. 16, no. 4, pp. 193-210, 2003.

[7] R. G. Brown, M. Jahanshahi, and C. D. Marsden, "Response choice in Parkinson's disease. The effects of uncertainty and stimulus-response compatibility," Brain, vol. 116, no. 4, pp. 869-885, 1993.

[8] A. E. Taylor and J. A. Saintcyr, "The neuropsychology of Parkinsons-disease," Brain and Cognition, vol. 28, no. 3, pp. 281-296, 1995.

[9] R. H. S. Mindham and T. A. Hughes, "Cognitive impairment in Parkinson's disease," International Review of Psychiatry, vol. 12, no. 4, pp. 281-289, 2000.

[10] J. Green, W. M. McDonald, J. L. Vitek et al., "Cognitive impairments in advanced PD without dementia," Neurology, vol. 59, no. 9, pp. 1320-1324, 2002.

[11] A. Costa, A. Peppe, G. Dell'Agnello et al., "Dopaminergic modulation of visual-spatial working memory in Parkinson's disease," Dementia and Geriatric Cognitive Disorders, vol. 15, no. 2, pp. 55-66, 2003.

[12] S. M. L. Cox, E. Stefanova, I. S. Johnsrude, T. W. Robbins, and A. M. Owen, "Preference formation and working memory in Parkinson's disease and normal ageing," Neuropsychologia, vol. 40, no. 3, pp. 317-326, 2002.

[13] P. S. Foster, V. Drago, G. P. Crucian et al., "Verbal and visuospatial memory in lateral onset Parkinson disease: time is of the essence," Cognitive and Behavioral Neurology, vol. 23, no. 1, pp. 19-25, 2010.

[14] J. V. Filoteo and W. T. Maddox, "Quantitative modeling of visual attention processes in patients with Parkinson's disease: effects of stimulus integrality on selective attention and dimensional integration," Neuropsychology, vol. 13, no. 2, pp. 206-222, 1999.

[15] C. L. Bras, B. Pillon, P. Damier, and B. Dubois, "At which steps of spatial working memory processing do striatofrontal circuits intervene in humans?" Neuropsychologia, vol. 37, no. 1, pp. 83-90, 1998.

[16] J. D. E. Gabrieli, J. Singh, G. T. Stebbins, and C. G. Goetz, "Reduced working memory span in Parkinson's disease: evidence for the role of a frontostriatal system in working and strategic memory," Neuropsychology, vol. 10, no. 3, pp. 322332, 1996.

[17] M. L. Waterfall and S. F. Crowe, "Meta-analytic comparison of the components of visual cognition in Parkinson's disease," Journal of Clinical and Experimental Neuropsychology, vol. 17, no. 5, pp. 759-772, 1995. 
[18] L. Gueye, F. Viallet, E. Legallet, and E. Trouche, "The use of advance information for motor preparation in parkinson's disease: effects of cueing and compatibility between warning and imperative stimuli," Brain and Cognition, vol. 38, no. 1, pp. 66-86, 1998.

[19] N. Georgiou, J. L. Bradshaw, R. Iansek, J. G. Phillips, J. B. Mattingley, and J. A. Bradshaw, "Reduction in external cues and movement sequencing in Parkinson's disease," Journal of Neurology Neurosurgery and Psychiatry, vol. 57, no. 3, pp. 368370, 1994.

[20] N. Georgiou, R. Iansek, J. L. Bradshaw, J. G. Phillips, J. B. Mattingley, and J. A. Bradshaw, "An evaluation of the role of internal cues in the pathogenesis of Parkinsonian hypokinesia," Brain, vol. 116, no. 6, pp. 1575-1587, 1993.

[21] M. J. Majsak, T. Kaminski, A. M. Gentile, and J. R. Flanagan, "The reaching movements of patients with Parkinson's disease under self-determined maximal speed and visually cued conditions," Brain, vol. 121, no. 4, pp. 755-766, 1998.

[22] Q. J. Almeida, L. R. Wishart, and T. D. Lee, "Disruptive influences of a cued voluntary shift on coordinated movement in Parkinson's disease," Neuropsychologia, vol. 41, no. 4, pp. 442-452, 2003.

[23] R. G. Brown and C. D. Marsden, "An investigation of the phenomenon of "set" in Parkinson's disease," Movement Disorders, vol. 3, no. 2, pp. 152-161, 1988.

[24] R. G. Brown and C. D. Marsden, "Internal versus external cues and the control of attention in Parkinson's disease," Brain, vol. 111, no. 2, pp. 323-345, 1988.

[25] J. L. Bradshaw, M. L. Waterfall, J. G. Phillips, R. Iansek, J. B. Mattingley, and J. A. Bradshaw, "Re-orientation of attention in Parkinson's disease: an extension to the vibrotactile modality," Neuropsychologia, vol. 31, no. 1, pp. 51-66, 1993.

[26] R. Cunnington, R. Iansek, and J. L. Bradshaw, "Movementrelated potentials in Parkinson's disease: external cues and attentional strategies," Movement Disorders, vol. 14, no. 1, pp. 63-68, 1999.

[27] J. P. Azulay, S. Mesure, and O. Blin, "Influence of visual cues on gait in Parkinson's disease: contribution to attention or sensory dependence?" Journal of the Neurological Sciences, vol. 248, no. 1-2, pp. 192-195, 2006.

[28] K. Baker, L. Rochester, and A. Nieuwboer, "The immediate effect of attentional, auditory, and a combined cue strategy on gait during single and dual tasks in Parkinson's disease," Archives of Physical Medicine and Rehabilitation, vol. 88, no. 12, pp. 1593-1600, 2007.

[29] K. Baker, L. Rochester, and A. Nieuwboer, "The effect of cues on gait variability-Reducing the attentional cost of walking in people with Parkinson's disease," Parkinsonism and Related Disorders, vol. 14, no. 4, pp. 314-320, 2008.

[30] M. Jahanshahi, R. G. Brown, and C. D. Marsden, "Simple and choice reaction time and the use of advance information for motor preparation in Parkinson's disease," Brain, vol. 115, no. 2, pp. 539-564, 1992.

[31] S. Ledger, R. Galvin, D. Lynch, and E. K. Stokes, "A randomised controlled trial evaluating the effect of an individual auditory cueing device on freezing and gait speed in people with Parkinson's disease," BMC Neurology, vol. 8, article 46, 2008.

[32] I. Lim, E. van Wegen, C. de Goede et al., "Effects of external rhythmical cueing on gait in patients with Parkinson's disease: a systematic review," Clinical Rehabilitation, vol. 19, no. 7, pp. 695-713, 2005.

[33] A. Nieuwboer, G. Kwakkel, L. Rochester et al., "Cueing training in the home improves gait-related mobility in Parkinson's disease: the RESCUE trial," Journal of Neurology, Neurosurgery and Psychiatry, vol. 78, no. 2, pp. 134-140, 2007.
[34] E. van Wegen, C. de Goede, I. Lim et al., "The effect of rhythmic somatosensory cueing on gait in patients with Parkinson's disease," Journal of the Neurological Sciences, vol. 248, no. 1-2, pp. 210-214, 2006.

[35] E. Wascher, R. Verleger, P. Vieregge, P. Jaskowski, S. Koch, and D. Kömpf, "Responses to cued signals in Parkinson's disease. Distinguishing between disorders of cognition and of activation," Brain, vol. 120, no. 8, pp. 1355-1375, 1997.

[36] E. Seiss and P. Praamstra, "The basal ganglia and inhibitory mechanisms in response selection: evidence from subliminal priming of motor responses in Parkinson's disease," Brain, vol. 127, no. 2, pp. 330-339, 2004.

[37] P. Praamstra, D. F. Stegeman, A. R. Cools, and M. W. I. M. Horstink, "Reliance on external cues for movement initiation in Parkinson's disease. Evidence from movementrelated potentials," Brain, vol. 121, no. 1, pp. 167-177, 1998.

[38] T. Akamatsu, H. Fukuyama, and T. Kawamata, "The effects of visual, auditory, and mixed cues on choice reaction in Parkinson's disease," Journal of the Neurological Sciences, vol. 269, no. 1-2, pp. 118-125, 2008.

[39] J. Fielding, N. Georgiou-Karistianis, L. Millist, and O. White, "Temporal variation in the control of goal-directed visuospatial attention in basal ganglia disorders," Neuroscience Research, vol. 54, no. 1, pp. 57-65, 2006.

[40] K. A. Briand, W. Hening, H. Poizner, and A. B. Sereno, "Automatic orienting of visuospatial attention in Parkinson's disease," Neuropsychologia, vol. 39, no. 11, pp. 1240-1249, 2001.

[41] P. M.J. Pollux and C. Robertson, "Voluntary and automatic visual spatial shifts of attention in Parkinson's disease: an analysis of costs and benefits," Journal of Clinical and Experimental Neuropsychology, vol. 23, no. 5, pp. 662-670, 2001.

[42] M. Rizzo, E. Y. Uc, J. Dawson, S. Anderson, and R. Rodnitzky, "Driving difficulties in Parkinson's disease," Movement Disorders, vol. 25, no. 1, pp. S136-140, 2010.

[43] E. I. Klimkeit, J. L. Bradshaw, J. Charlton, R. Stolwyk, and N. Georgiou-Karistianis, "Driving ability in Parkinson's disease: current status of research," Neuroscience and Biobehavioral Reviews, vol. 33, no. 3, pp. 223-231, 2009.

[44] J. A. Groeger, "Understanding driving: applying cognitive psychology to a complex everyday task," in Frontiers of Cognitive Science, Psychology Press, New York, NY, USA, 2000.

[45] S. G. Charlton, "Perceptual and attentional effects on drivers' speed selection at curves," Accident Analysis and Prevention, vol. 36, no. 5, pp. 877-884, 2004.

[46] H. Walter, S. C. Vetter, JO. Grothe, A. P. Wunderlich, S. Hahn, and M. Spitzer, "The neural correlates of driving," NeuroReport, vol. 12, no. 8, pp. 1763-1767, 2001.

[47] E. Y. Uc, M. Rizzo, S. W. Anderson, J. D. Sparks, R. L. Rodnitzky, and J. D. Dawson, "Driving with distraction in Parkinson disease," Neurology, vol. 67, no. 10, pp. 1774-1780, 2006.

[48] J. Grace, M. M. Amick, A. D’abreu, E. K. Festa, W. C. Heidel, and B. R. Ott, "Neuropsychological deficits associated with driving performance in Parkinson's and Alzheimer's disease," Journal of the International Neuropsychological Society, vol. 11, no. 6, pp. 766-775, 2005.

[49] V. M. Heikkila, J. Turkka, J. Korpelainen, T. Kallanranta, and H. Summala, "Decreased driving ability in people with Parkinson's disease," Journal of Neurology Neurosurgery and Psychiatry, vol. 64, no. 3, pp. 325-330, 1998.

[50] K. A. Radford, N. B. Lincoln, and G. Lennox, "The effects of cognitive abilities on driving in people with Parkinson's disease," Disability and Rehabilitation, vol. 26, no. 2, pp. 6570, 2004. 
[51] J. M. Wood, C. Worringham, G. Kerr, K. Mallon, and P. Silburn, "Quantitative assessment of driving performance in Parkinson's disease," Journal of Neurology, Neurosurgery and Psychiatry, vol. 76, no. 2, pp. 176-180, 2005.

[52] R. Cordell, H. C. Lee, A. Granger, B. Vieira, and A. H. Lee, "Driving assessment in Parkinson's disease-a novel predictor of performance?" Movement Disorders, vol. 23, no. 9, pp. 1217-1222, 2008.

[53] S. Classen, D. P. McCarthy, O. Shechtman et al., "Useful field of view as a reliable screening measure of driving performance in people with parkinson's disease: results of a pilot study," Traffic Injury Prevention, vol. 10, no. 6, pp. 593-598, 2009.

[54] E. Y. Uc, M. Rizzo, S. W. Anderson, J. Sparks, R. L. Rodnitzky, and J. D. Dawson, "Impaired visual search in drivers with Parkinson's disease," Annals of Neurology, vol. 60, no. 4, pp. 407-413, 2006.

[55] E. Y. Uc, M. Rizzo, S. W. Anderson, J. D. Sparks, R. L. Rodnitzky, and J. D. Dawson, "Impaired navigation in drivers with Parkinson's disease,” Brain, vol. 130, no. 9, pp. 2433 2440, 2007.

[56] P. Madeley, J. L. Hulley, H. Wildgust, and R. H. S. Mindham, "Parkinson's disease and driving ability," Journal of Neurology Neurosurgery and Psychiatry, vol. 53, no. 7, pp. 580-582, 1990.

[57] R. J. Stolwyk, T. J. Triggs, J. L. Charlton, R. Iansek, and J. L. Bradshaw, "Impact of internal versus external cueing on driving performace in people with Parkinson's disease," Movement Disorders, vol. 20, no. 7, pp. 846-857, 2005.

[58] S. Lings and E. Dupont, "Driving with Parkinson's disease. A controlled laboratory investigation," Acta Neurologica Scandinavica, vol. 86, no. 1, pp. 33-39, 1992.

[59] L. M. Vaux, R. Ni, M. Rizzo, E. Y. Uc, and G. J. Andersen, "Detection of imminent collisions by drivers with Alzheimer's disease and Parkinson's disease: a preliminary study," Accident Analysis and Prevention, vol. 42, no. 3, pp. 852-858, 2010.

[60] T. A. Zesiewicz, C. R. Cimino, A. R. Malek et al., "Driving safety in Parkinson's disease," Neurology, vol. 59, no. 11, pp. 1787-1788, 2002.

[61] M. F. Folstein, S. E. Folstein, and P. R. McHugh, ““"Mini mental state". A practical method for grading the cognitive state of patients for the clinician," Journal of Psychiatric Research, vol. 12, no. 3, pp. 189-198, 1975.

[62] S. T. Godley, T. J. Triggs, and B. N. Fildes, "Driving simulator validation for speed research," Accident Analysis and Prevention, vol. 34, no. 5, pp. 589-600, 2002.

[63] M. Jahanshahi, R. G. Brown, and C. D. Marsden, "The effect of withdrawal of dopaminergic medication on simple and choice reaction time and the use of advance information in Parkinson's disease," Journal of Neurology Neurosurgery and Psychiatry, vol. 55, no. 12, pp. 1168-1176, 1992.

[64] R. J. Stolwyk, J. L. Charlton, T. J. Triggs, R. Iansek, and J. L. Bradshaw, "Neuropsychological function and driving ability in people with Parkinson's disease," Journal of Clinical and Experimental Neuropsychology, vol. 28, no. 6, pp. 898-913, 2006.

[65] M. M. Amick, J. Grace, and B. R. Ott, "Visual and cognitive predictors of driving safety in Parkinson's disease patients," Archives of Clinical Neuropsychology, vol. 22, no. 8, pp. 957967, 2007.

[66] E. Y. Uc, M. Rizzo, S. W. Anderson, E. Dastrup, J. D. Sparks, and J. D. Dawson, "Driving under low-contrast visibility conditions in Parkinson disease," Neurology, vol. 73, no. 14, pp. 1103-1110, 2009.
[67] C. J. Worringham, J. M. Wood, G. K. Kerr, and P. A. Silburn, "Predictors of driving assessment outcome in Parkinson's disease," Movement Disorders, vol. 21, no. 2, pp. 230-235, 2006. 


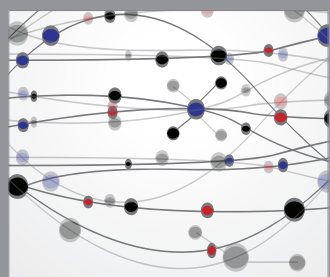

The Scientific World Journal
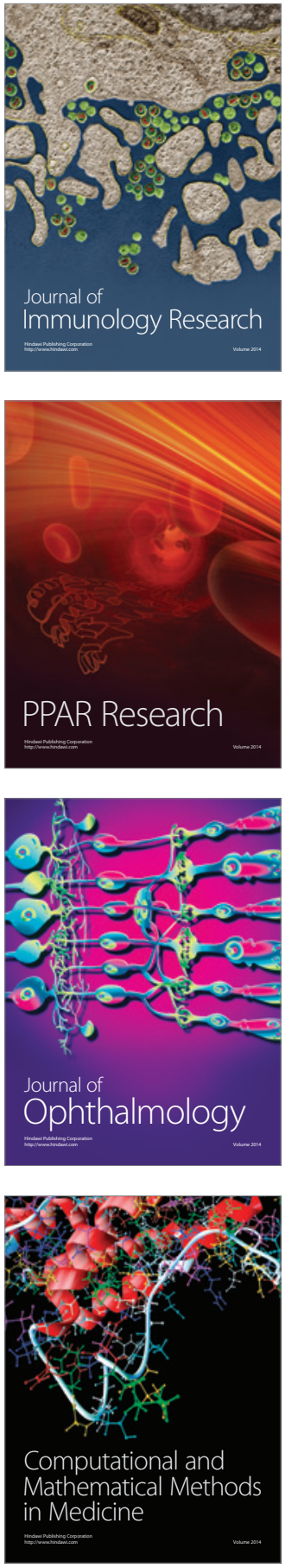

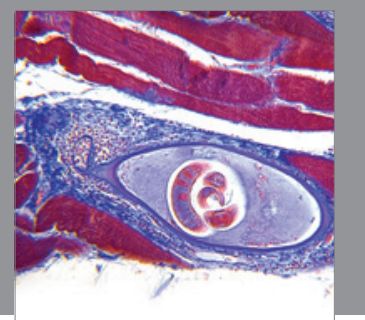

Gastroenterology

Research and Practice
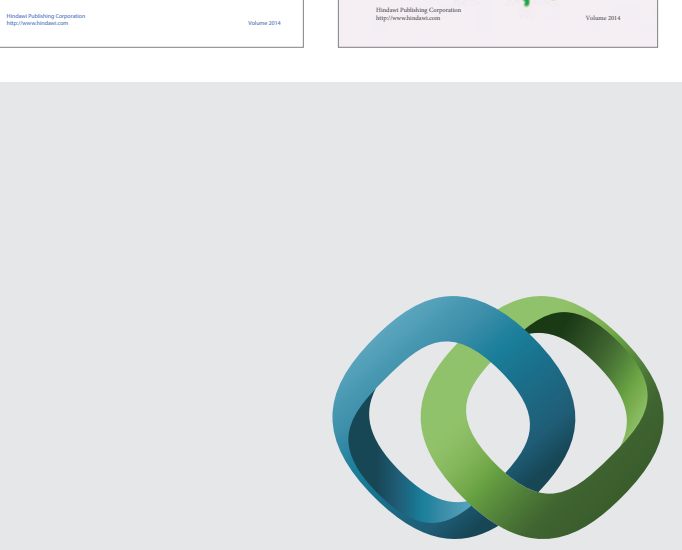

\section{Hindawi}

Submit your manuscripts at

http://www.hindawi.com
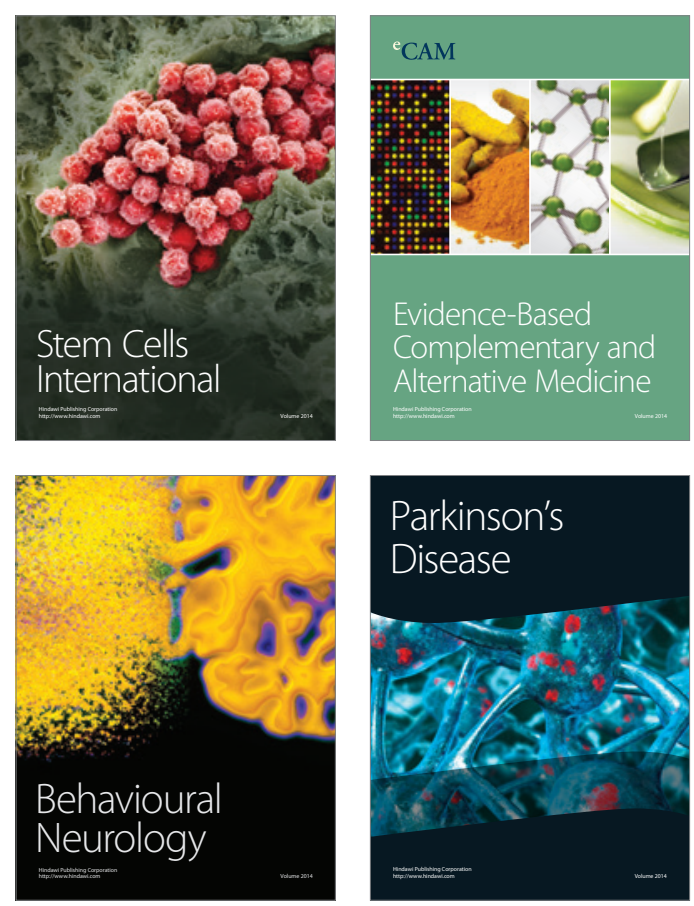

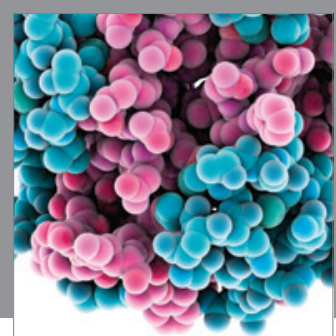

Journal of
Diabetes Research

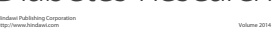

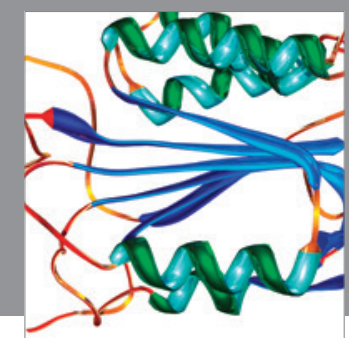

Disease Markers
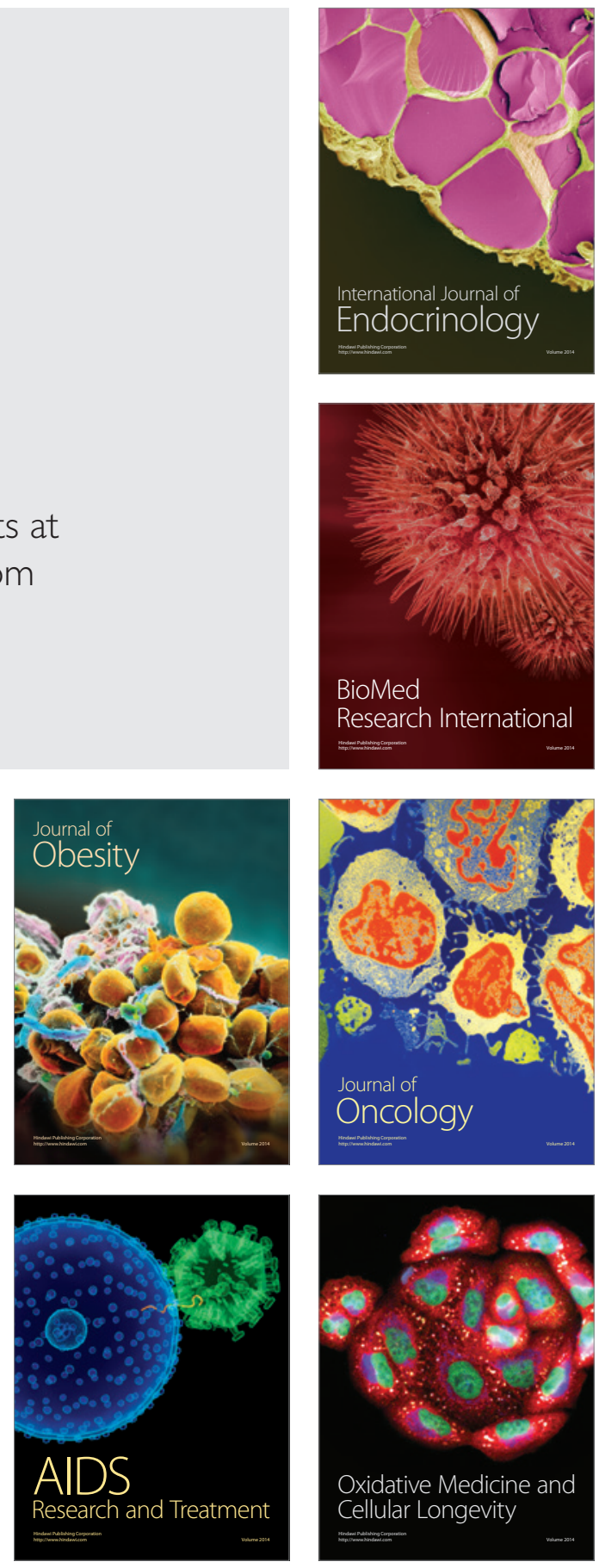